\title{
Optical biosensors based on the surface plasmon resonance phenomenon: optimization of the metal layer parameters
}

\author{
B.A. Snopok, K.V. Kostyukevich, S.I. Lysenko, P.M. Lytvyn, O.S. Lytvyn, \\ S.V. Mamykin, S.A. Zynyo, P.E. Shepeliavyi, S.A. Kostyukevich, Yu.M. Shirshov, E.F. Venger \\ Institute of Semiconductor Physics NAS of Ukraine, 45 prospect Nauki, 03028 Kyiv, Ukraine
}

\begin{abstract}
Application of the evanescent wave phenomena (e.g. surface plasmon resonance) in the chemical and biochemical sensors provides both optimal conditions for registration of specific interactions and high sensitivity. At the same time, the stability and reproducibility of parameters of a thin gold film (where the plasmon oscillations occur) are key problems for both $(i)$ optimal transformation of biochemical information into the signal that is convenient for further treatment and (ii) the formation of artificial interfacial architectures. The paper presents results concerning the study of the deposition process temperature regimes influence on optical properties and structure of thin gold films. Morphological and topographic features of the thin film structures were analyzed using X-ray diffraction and atomic force microscopy, respectively. It is shown that the simple procedure of the low temperature annealing $\left(\right.$ at $\sim 120^{\circ} \mathrm{C}$ ) provides optimum parameters of thin gold films in view of their application as physical transducers in optical biosensors. Finally, the SPR transducer was fabricated with high sensitive elements used in the device «PLASMON-4M» elaborated in the Institute of Semiconductor Physics (NAS of Ukraine).
\end{abstract}

Keywords: optical biosensor, surface plasmon resonance, thin gold layers, AFM, X-ray, optical properties, low-temperature annealing.

Paper received 29.01.01; revised manuscript received 14.02.01; accepted for publication 16.02.01.

\section{Introduction}

Analysis of the recent development of chemical and biochemical sensors evidences that in their design and construction the principles inherent to living beings are used more and more widely [1]. Therefore, the scientific and technological problems of utmost importance are those related to the development of sensitive elements and methods for the registration of specific interactions that take into account such peculiarities of molecular elements of living beings as lability, high sensitivity to strong electric and magnetic fields, importance of organic membranes for nondestructive immobilization, etc.

Application of the optoelectronic effects [2] for the registration of specific intermolecular interaction provides both optimal conditions for biological molecules (in view of the minimization of external influence) and high sensitivity. Indeed, conditions of surface plasmons excitation at the interface between thin gold film and solution under investigation (the effect of the surface plasmon resonance, SPR) depend on small variations of the optical constants of the media in the vicinity of the surface [3]. Processes of the intermolecular interaction cause the shift of the minimum of the excitation angle of the polariton states; the changes take place due the binding (or releasing) the molecules from the solutions with the receptor centers that are immobilized on the surface of an optoelectronic transducer [4]. In this case, the stability and reproducibility of the parameters of the thin gold film (where the plasmon oscillations occur) deposited on the surface of the glass prism are the key problems both for reliable measurements and exact calculations of the optical constants and adsorbed layer thickness (and, hence, the concentration of the molecules under investigation) using the experimental data. Moreover, besides the optimization of the sensitivity, stability and reproducibility of the sensor, one also needs to provide the formation of self-assembled monolayers with given properties on the surface of the sensor [5].

Investigations of the processes of the formation of the spatially-organized structures of tens and hundreds angstroms scale are important for both fundamental research 


\section{B.A. Snopok et al.: Optical biosensors based on the surface plasmon resonance}

and applications, because they are the base for the development of new technologies in microelectronics, heterogenic catalysis, sensors production [6,7]. It is obvious that the possibility to form the above self-assembled monolayers with given chemical functionality, which are responsible for the specifity of the sensor, depends mainly on fulfilment of the conditions for self-organizing molecular ensembles on the surface of solids [8]. Previously we have shown [9] for the case of the polycrystalline gold surface modification with aliphatic thiols that the simple process of thermal annealing can change the surface topography in such a way that self-organizing process provides the monolayer with close packing. At the same time, the influence of the temperature regimes of thin gold film fabrication on the sensitivity, stability and other characteristics of physical transducers has not been investigated.

Therefore, the present paper deals with the study of the influence of temperature regimes of a deposition process on optical, topographic and morphologic properties of thin gold films in order to improve the sensitivity, reproducibility and stability of SPR physical tranducers.

\section{Optical biosensors based on the effect of surface plasmon resonance}

Biosensor is the system that transforms information on the process of biochemical interaction of the analyte and the system of recepting centers immobilized on the surface of the physical transducer into the signal that is convenient for further treatment [10]. Usually, it is supposed that recepting centers have biological origin or are the artificial analogues of the biological ones [11]. Thus, the biosensor system has three principal components, namely:

- the physical transducer (PT) itself that provides high sensitivity and does not influence the process of the interaction between the receptor and analyte;

- sensing layer with immobilized recepting centers that are responsible for the specifity of the interaction with the given molecules in the solution;

- system of the preparation, transporting and disposing of the samples together with the devices of the analog and digital treatment of the output signal of the physical transducer that should provide stable composition and conditions during the measurements as well as related calibrating procedures and should display information on the process of the intermolecular interaction in acceptable form.

Methods of fabrication, purification and immobilization of recepting centers that have biological origin are widely described in biochemical papers [12-14] as well as measurements techniques and a necessary equipment [15-17]. At the same time, optimization of the physical transducers, especially for biosensor applications, remains a challenge in many aspects. Taking into account that the gold film is the medium for plasmon oscillations, on the one hand, and the substrate for the recepting centers immobilization, on the other hand, there are two directions of the physical transducers optimization, namely:
- the aim of the «physical» direction is to provide the most efficient transformation of the changes in the sensitive layer into the output signal; the efficiency is determined by both optical, and structural (topological and morphological) properties of the gold film;

- the aim of the «chemical» direction is to create the conditions for the oriented and nondestructive immobilization of the recepting centers;

The «chemical» optimization has two principal aspects: i) the topological one that controls the possibility of organized structures formation on the rough surface [6-8] and ii) the protecting aspect that prevents the denaturation of biomolecules on the metallic surface [18]. Possible ways of this optimization have been discussed by us earlier [9]. Therefore, we will study the «physical» optimization of the sensors in SPR transducer, i.e. of the thin gold film, for biological applications.

\subsection{Some aspects of the theory of elementary excitations of the surface}

The presence of the interface $(x y)$ between the crystal $(M)$ and the surrounding medium $(D)$ causes the appearance of the collective excitations that are localized on the surface; these excitations are related to the peculiarities of the electron and phonon spectra of the system as a whole. In metals (Au, Ag, etc.) the coherent superposition of electron-hole pairs forms the wave-like excitation of the charge density on the surface that is called the surface plasmon [19]. Similarly to its bulk analogue - the quasiparticle that describes the vibrations of electrons around heavy ions in the «electron plasma» of a solid - the surface plasmon is the longitudinal mode and its wavevector characterizes the «increase» and «decrease» of the charge density on the surface (this excitation is similar to the standing wave) [20]. The electric field of this excitation decreases exponentially with the distance from the surface [21]

$$
\vec{E}=\vec{E}_{0} \exp \left[i\left(\vec{k}_{x} \vec{x}+\vec{k}_{z} \vec{z}-\omega t\right)\right]
$$

where $\vec{k}_{x}$ is the wavevector in the direction of the plasmon propagation $(x), \vec{k}_{z}$ is the wave vector in the direction perpendicular to the interface $(z), \omega$ is the frequency, $t$ is time. The tangential component of the electric field is continuous $\left(k_{x M}=k_{x D}=k_{x}\right)$. The normal component has a discontinuity at $z=0$ and, taking into account the boundary conditions, the non-trivial solution occurs only at

$$
\frac{k_{z M}}{k_{z D}}=-\frac{\varepsilon_{M}}{\varepsilon_{D}}
$$

Consequently, surface polariton states can be excited only at the interface between the media with the dielectric constants of opposite signs. For the interface between a metal (gold) and a dielectric medium with positive dielectric constant (air, water solutions) the condition (2) is fulfilled for the phonons in the region of the visible light 


\section{B.A. Snopok et al.: Optical biosensors based on the surface plasmon resonance}

wavelengths that leads to the generation of the phonon surface polariton at the interface. Thus, in the macroscopic approximation, there is a surface charged layer on the flat interface $(z=0)$; the charge density in this layer is given by the difference of the $z$-components of electric induction vectors $\vec{D}$ in the media on the both sides of the interface:

$$
\left(\vec{D}_{D}-\vec{D}_{M}\right) \vec{z}=4 \pi \sigma
$$

The mechanism of the surface plasmon excitation is probably caused by different character and decrease of the electric field in metal and surrounding medium; as a consequence, the prevailing part of the energy flux of the wave is concentrated above the surface of the solid.

It is known that normal modes do not directly interact with the electromagnetic wave because their dispersion region does not coincide with the frequency range where the energy and impulse conservation laws can be simultaneously fulfilled. Thus, the incident electromagnetic wave can not directly excite a plasmon resonance at any angle. There are two main mechanisms that relate the incident radiation with polaritons. The first one is the direct interaction of the incident light with polariton due to diffraction of the incident light on the rough surface; the latter causes the increase of the longitudinal impulse [22]. The second mechanism is the direct consequence of the conservation laws [23]. In fact, the energy and impulse conservation laws at the surface plasmons interaction with light, on the one hand, and the shape of the dispersion curves, on the other hand, do not contradict each other only in the case when the light wave is characterized by the imaginary $k_{z}$ value [21]. This inhomogeneous wave with the imaginary component of the wave vector that is perpendicular to the interface $(z)$ emerges at the total internal reflection on the interface between more and less dense media. This wave propagates along the interface in the incidence plane and exponentially decreases towards the less optically dense medium at the distance of about wavelength. Therefore, the most convenient method to generate the surface polaritons in metals is the plasmon resonance method that is one of the modifications of the total internal reflection (TIR) method. The most widely used modification of the TIR method is Kretschmann's configuration [24] mostly due to its experimetal availability and reliability of the results. In this case the photons with large impulse in prism ( $k^{p h}=\frac{\omega}{c} \sqrt{\varepsilon_{p}}$, where $\varepsilon_{p}$ is the dielectric constant of the prism) interact with the polaritonic states through the thin metal layer deposited directly on the base of glass prism [18].

The surface plasmon resonance causes a sharp minimum on the curve of the reflection coefficient for the $p$ polarized light. Its position $\theta_{S P R}$, half-width $\Delta R$ and the depth of the minimum $R_{\text {min }}$ are determined by both the parameters of the metal layer and optical constants of the surrounding medium. The expressions for the wavevectors of the plasmon in the dielectric medium are [21]: $k_{x}=\frac{\omega}{c} \sqrt{\varepsilon_{D}\left(\frac{\omega}{c}\right)^{2}-k_{x}^{2}} \quad k_{z D}=\sqrt{\varepsilon_{D}\left(\frac{\omega}{c}\right)^{2}-k_{x}^{2}}$

It should be noted that both $\varepsilon_{M}$ and complex refractive index $\left(\tilde{n}=n_{M}+i k_{M}\right)$ are the complex values and, therefore, $k_{x}$ is also the complex value, i.e. $k_{x}=k_{x}+i k_{x}$. The characteristic length of the plasmon propagation along the surface $L_{x}$ can be estimated using the expression $L_{x}=1 / k_{x}^{\prime \prime}$. Moreover, according to (1) the surface plasmon is the bound nonradiative mode with the field amplitude being maximum on the surface $(z=0)$ and exponentially decreasing on both sides of the interface. The penetration depth in the dielectric medium is about several hundreds of nanometers; this is the reason why the SPR is so selective and highly sensitive especially to surface processes. General expressions for the SPR curves can be found in [25]. Hereafter we will discuss only the principal dependencies of the SPR curves on the metal layer characteristics.

The results of the numerical simulation of SPR curves [26] at different values of optical constants and metal layer thicknesses lead us to several important conclusions: i) the position of the minimum on the SPR curve for a clean metallic surface does not depend (in the first approximation) on the thickness $d_{M}$ and real part of the complex refractive index $n_{M}$ of the metal layer; at the same conditions it is determined by the metal absorption coefficient value: the less $k_{M}$, the larger are $\theta_{S P R}$ and $\Delta R$; ii) the thickness of the metal layer $d_{M}$ and the real part of the refractive index of the metal layer influence mostly the $R_{\min }$ value. The peculiar feature is the variation of the SPR curves in the region of the transition to the TIR and at large angles (the larger is $n_{M}$, the smaller is the intensity of the reflection). At the same time, the variation of the layer thickness essentially influences the $R_{\text {min }}$ value that increases when the layer thickness differs from the optimum value. As these parameters $\left(d_{M}\right.$ and $n_{M}$ ) are mutually dependent, one needs additional information to avoid ambiguity in the calculated values of $d_{M}$ and $n_{M}$. Thus, to determine true optical parameters and thin metal layer thicknesses using SPR one should analyze the whole plasmon resonance curve using numerical approximation of the experimental curve with theoretical expression in accordance with the complete mathematical description of the optical system. Note, that Abel method [27] and John's matrices of scattering formalism [28] are the most adequate methods for the numerical simulation of the above systems.

\subsection{Effects of the structure and parameters of the metal film on parameters of SPR curves in the Kretschmann geometry}

As it was emphasized above, in the most widely used geometry of SPR investigation - the Kretschmann method the incident and reflected beams come through the metal 


\section{B.A. Snopok et al.: Optical biosensors based on the surface plasmon resonance}

layer that is the medium for plasmon vibrations. To increase the signal-to-noise ratio the thickness of the metal film is chosen in such a way that $R_{\text {min }} \approx 0$. The corresponding value of $d_{M}$ can be estimated using numerical calculations [33] and experimentally determined by standard optical techniques using wedge-like metal film [29]. It is supposed that metal film has homogeneous structure as well as plane and parallel surfaces. It is clear that the real situation differs from the above idealized model and the influence of the film structure on the SPR curves parameters should be taken into account.

The measured value of the reflection coefficient in the system prism/film/surrounding medium is determined by the following mechanisms:

- surface plasmon excitation. In this case the SPR curve should be described by the model of the metal layer with plane and parallel surfaces with optical parameters of single crystalline samples;

- scattering and/or absorption on the inner inhomogeneities (defects, pores, crystallite boundaries, impurities, etc.), that cause the fluctuations of dielectric susceptibility of the film. Exact account of these processes is very complicated because the above effects can influence on the values $\theta_{S P R}, \Delta R$ and $R_{\min }$ in different ways and nonlinearly depend on the angle. To diminish the influence of the internal inhomogeneity of the film on the SPR curve one should fabricate the films with maximum isotropy and minimum contribution of polycrystalline phase. Another important point is to prevent the changes of the film parameters with time («aging») or under the influence of the surrounding medium. In any case, if the film thickness $d_{m}$ have been chosen so that one gets minimum $R_{\min }$ then the internal inhomogeneity causes the increase of $R_{\min }$ and $\Delta R$;

- influence of the roughness. Influence of the surface relief on the SPR curve is, probably, the most essential factor that causes changes in light extintion due to the presence of high electric field just on the film surface. The surface plasmon propagating in certain direction, scatters and changes its direction. The fields related to such plasmon, naturally, have smaller phase velocity. Moreover, the surface waves decays when spreading along the rough surface. It should be noted that damping caused by the radiation of light is forbidden on the smooth surface [30]. These processes lead to the following behavior of the minimum reflection vs. roughness function: i) resonance shifts towards larger values at a fixed wavelength of the incident light; ii) the width of the dip in the reflection spectrum $\Delta R$ increases with roughness.

\subsection{The ways for SPR optoelectronic trans- ducer optimization}

In the case of PTs that use the effect of the surface plasmon resonance, the physical parameter measured during the receptor/analyte reaction is the variation of the refraction index $\Delta n=n-n_{0}$ of the reactive layer of the thickness $d$; using this value the surface concentration $\Gamma$ can be easily obtained in accordance with the classical approach proposed by de Feijter [31]:

$\Gamma=\frac{d\left(n-n_{0}\right)}{d n / d c}$

where the value $d n / d c$ is about $0.188 \mathrm{~cm}^{3} / \mathrm{g}$ for most biological molecules [32].

This variation causes the shift of the maximum of the TIR curve $\theta_{S P R}$, which corresponds to the most full transformation of the energy of incident wave into surface polariton state at given conditions. The efficiency of the PT is related to the degree of the transformation of the biochemical information about the process of the receptor/analyte interaction into the corresponding output signal. Thus, the efficiency of the SPR based PT in the linear range, i.e., when $d<<$ wavelength of polaritons, will be characterized by the transformation constant $K$ that is determined as

$K=\frac{\Delta \theta_{S P R}}{\Delta n \cdot d}$

Dependence of $\mathrm{K}$ on the optical constants of the gold film in the case of its optimum thickness $(45 \mathrm{~nm})$ is shown in Fig. 1. As it is seen, the variation of the absolute value of the metal film refractive index almost does not influence $K$, while the variation of the absorption coefficient leads to the exponential dependence. The smaller is the absolute value of the absorption coefficient, the larger is the constant $K$. Thus, to increase the sensitivity of the method one should try to diminish the imaginary part of the film refractive index. The increase of SPR curve width does not influence essentially the accuracy of the minimum value $\theta_{S P R}$ (that is determined from the polynomial approximation of the experimental dependencies) and the

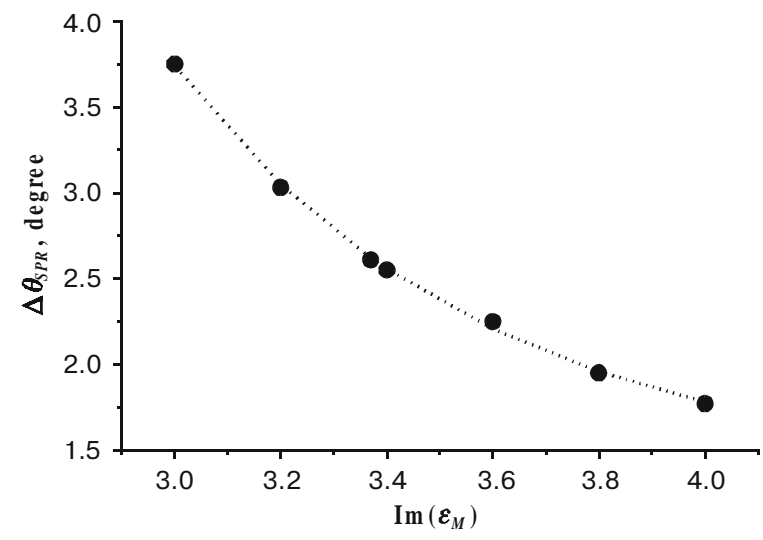

Fig. 1. Simulated set of the shift of the SPR angle $\left(\Delta \theta_{s p r}=\theta_{s p r}\right.$ sample $\left.-\theta_{\text {spr }}{ }^{\text {gold }}\right)$ versus the deviation of the permittivity imaginary part $\operatorname{Im}\left(\varepsilon_{M}\right)$ of gold film. The sensor chip was represented by a model system consisting of the semi-infinite glass prism $\left(n_{p}=1.515+0 \cdot i\right)$, gold film $\left(n_{A u}=0.16+3.37 \cdot i\right.$, thickness $\left.45 \mathrm{~nm}\right)$, adlayer $\left(n_{s}=1.337+\right.$ $+0 \cdot i, d=10 \mathrm{~nm})$ and semi-infinite buffer solution $\left(n_{b}=1.337+0 \cdot i\right)$. The dotted line is the best fit for simulated data: $\Delta \theta_{s p r}=1.35+$ $\left.+2.39 \exp \left(-\left(\operatorname{Im}\left(\varepsilon_{M}\right)-3\right) / 0.59\right)\right)$. 


\section{B.A. Snopok et al.: Optical biosensors based on the surface plasmon resonance}

shift of the SPR curves towards large angles can be easily compensated by adjusting the prism angle.

Since both incident and reflected beams in the Kretschmann geometry cross the metal film where the plasmon vibrations occur, the influence of the metal structure on the shape of the plasmon resonance curve is very important. In fact, absorption and scattering both in the bulk of the film and on the interfaces can diminish the signalto-noise ratio in the system [33]. In this case to find the minimum of reflection from the system prism/gold film/ surrounding medium one should carry out the approximation at the lowest absolute values of the reflected signal; therefore, the signal variations related to any other factors than polariton states can diminish the accuracy of approximation. Consequently, to decrease the scattering of light both in the bulk and on the interface is the important technological task.

The additional parameter that influences the longterm operation of the physical transducer is the time stability of its parameters. In accordance with [34], the instability of the as-deposited gold films is caused by mechanical strains that relax during several hours after the deposition at ambient conditions. Other authors report the long-time relaxation of both optical properties and surface topology [42]. Thus, the problem needs additional study.

Another consequence of the polycrystalline structure of the gold films obtained at standard conditions of deposition on the dielectric substrates with the roughness of nanometer scale covered with adhesive chromium layer is the possible penetration of the solutions into the bulk of the film along the boundaries of the grains. This process can cause both the changes of the optical parameters and chemical «aging» of the structure. Therefore, the films should have minimum number of pores to prevent the above processes. Probably, for the complete solution of this problem a chemical modification of the surface (e.g., by thiols ) should be carried out [9].

In conclusion, it should be noted that the technological processes that are used for the improvement of the above parameters must be chosen with the account of the specific features of SPR sensors (i.e. the temperatures should not exceed $300^{\circ} \mathrm{C}$ in order not to damage the glass prisms, strong alkali solutions cannot be used because of possible adhesive $\mathrm{Cr}$ layer dissolution, etc.), should be compatible with the following steps of receptor layer formation, and should be cost effective.

\section{Experimental}

\subsection{Materials}

For the investigations we used the polished quartz $\left(n_{s}=\right.$ 1.46) substrates with the dimensions $41 \times 19 \times 1.5 \mathrm{~mm}^{3}$ with low mean square roughness of the surface $(c a .1 .1 \mathrm{~nm})$ that were thoroughlly cleaned chemically (in chromium acid with a subsequent rinsing in large amount of water in the ultrasonic bath) and treated in the glowing discharge just before deposition.
Gold films of 30-60 nm thickness were deposited in vacuum (VUP-4; residual vapor pressure $4 \cdot 10^{-4} \mathrm{~Pa}$ ) on the adhesive-improving chromium layer (thickness $c a$. $1 \mathrm{~nm})$. The temperature of the substrates at the deposition varied from 293 to $473 \mathrm{~K}$. Deposition rate was $40-50 \AA / \mathrm{s}$, because this value provides gold films with reproducible optical parameters and smooth homogenous surface, while the decrease of the deposition rate leads to the decrease of some crystallites at the simultaneous increase of the size dispersion. The samples deposited at $293 \mathrm{~K}$ were annealed in the temperature range $353-473 \mathrm{~K}$. During the deposition the film thickness was controlled using quartz thickness sensor (KIT-1); the thickness was measured using AFM measurements of the groove profile obtained by photolithography method using positive photoresist (AZ 1350) and the etchant for gold that contained: $5 \mathrm{~g}$ of potassium iodine, $2.5 \mathrm{~g}$ of crystalline iodine and $10 \mathrm{ml}$ of water.

\subsection{Methods}

Atomic Force Microscopy. Surface topography of the films was studied with atomic force microscope (Nanoscope IIIa, Digital Instrument, Santa-Barbara) with $80 \mu \mathrm{m}$ scanner. Surface images were obtained in tapping mode using $\mathrm{Si}_{3} \mathrm{~N}_{4}$ needles; the scanning frequency was about $1 \mathrm{~Hz}$. $X$-ray diffraction. X-ray diffraction studies were carried out using the modified two-crystal DRON-3M spectrometer. The germanium crystal-monochromator was used to obtain monochromatic component of the radiation of the tube with the copper anticathode radiation. As the thickness of the gold films is low $(\sim 100 \AA)$ the asymmetric diffraction scheme was used: the angle between the sample and incident $\mathrm{X}$-rays beam was $3^{\circ}$ and the spectrum was registered at detector scanning in the angle range from $0^{\circ}$ to $90^{\circ}$. This scheme of measurements permits to increase the intensity of the radiation scattered by the sample and, thus, to improve the accuracy. Surface Plasmon Resonance. Spectroscopy of the surface plasmon resonance was carried out in air in the Kretschmann configuration using goniometer (G5M), glass prism (angle $45^{\circ}, n_{p}=1.52$ ), glycerin as an immersion liquid $\left(n_{G}=1.45\right)$ and He-Ne laser $(\lambda=632.8 \mathrm{~nm})$ as a light source. The principles of SPR measurements and the installation are described in [36].

Transmission/Reflection Measurements. The transmission/reflection of thin gold films was measured using the installation consisting of the monochromator IKS-12, CDSH-100 lamp and silicon light detector FD-24K. The characteristics were measured at the angles $10^{\circ}, 50^{\circ}$ and $70^{\circ}$ as to normal incidence of light in the frequency range $0.45-1 \mu \mathrm{m}$; the dependence of the optical constants (refractive index, absorption coefficient) on the wavelength was taken into account. Calculations were carried out in the one-layer model. The experimental dependencies of the transmission/reflection at the above angles were approximated by theoretical curves calculated by the Fresnel formulae [37]. The gold film thickness values used were obtained from AFM measurements.

SQO, 4(1), 2001 


\section{B.A. Snopok et al.: Optical biosensors based on the surface plasmon resonance}

Angle Resolved Scattering. Measurements of the scattered light indicatrix were carried out using special installation described in details in [33]. In brief, the beam of the He-Ne laser $(632.8 \mathrm{~nm}, 2 \mathrm{~mW})$ was focused on the surface of the sample; the diameter of the spot was $100 \mu \mathrm{m}$. The normal incidence was used. The intensity of the scattered light was detected by the RMT photomultiplier that could be rotated around the measured point on the sample by step motors. The angular dependence of the scattered light intensity was normalized by the body angle of the photodetector, as well as by the intensity of the incident beam; the latter was measured using the reflecting standard silicon wafer and calibrated light filter. To change the measured point, the cell with the sample under investigation was moved with the $x y$-stage with the accuracy $\pm 10 \mu \mathrm{m}$.

\section{Results and discussion}

First of all we are going to analyze the transmission and reflection spectra in order to find out the influence of the thermal annealing on the effective optical parameters of thin gold films. Then we shall discuss the results of the morphological (X-ray diffraction) and topographic (AFM) investigations in view of their influence on the optical properties and stability of PT SPR. In conclusion we will discuss the peculiarities of thin film model and the ways to prevent the radiation losses in the course of surface polariton generation.

\subsection{Optical characteristics of thin gold films}

In spite of being widely investigated for more than one hundred years the problem of the correlation between the structure and optical characteristics of thin films is not solved yet. The reason is the absence of both the reliable methods of $n_{M}$ and $k_{M}$ determination in thin layers with adequate account of the contribution of surface effects and the clear model describing optical properties of spatially inhomogeneous (polycrystalline) films. Our analysis of literature data shows that the range of optical con-

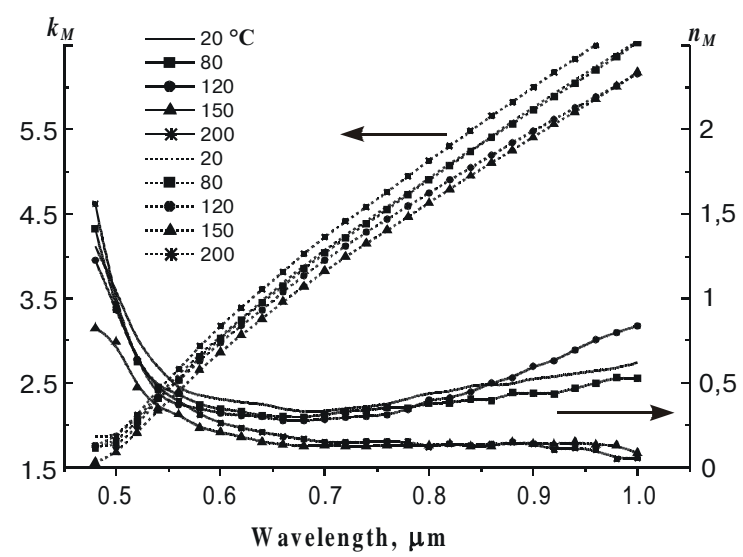

stants variation spreads up to $20 \%$ in the case of gold films [38-47]. Therefore, we took magnitudes 0.2 and 3.3 for $n_{M}$ and $k_{M}$, respectively, as the most reliable values. These were accepted hereafter as the parameters of the bulk phase for thin gold films used.

It should be also noted that in accordance with [46] the optical parameters of gold films depend on their thickness. The parameters that characterize the bulk phase are fairly reproducible only for the films with the thickness that does not exceed the critical one $\approx 250 \AA$ (thin ( $<200 \AA)$ gold films on the fused quartz substrates have spatially inhomogeneous and island structure). In this case the annealing of the films in nitrogen atmosphere at $150^{\circ} \mathrm{C}$ during $10-12$ hours does not cause any changes of optical parameters of the films.

\subsection{Transmission and reflection spectra of thin gold films}

The dependencies of the transmission $(T)$ and reflection $(R)$ of thin gold films on quartz substrates demonstrate the influence of both the regime and the thermal annealing conditions on the optical characteristics of gold films. Analysis of the data obtained leads to the following conclusions: i) maximum of the transmission occurs at $499 \mathrm{~nm}$ for hot substrates and at $519 \mathrm{~nm}$ for the samples deposited at $20^{\circ} \mathrm{C}$ and subsequently annealed; different positions of maxima can be explained by the difference in the preferable crystallite orientation; ii) the temperature causes the variation of $R$ as well as $T$, and its influence is the most pronounced particularly in the range of maximum $R$ and $T$ values. Thus, the increase of temperature does not cause the increase of transmission that could be expected in view of diminishing of the scattering on the inhomogeneities in the films structure. This fact evidences that the low temperature annealing is a complicated process that includes several different processes. It should be noted that in the wavelengths range $\sim 550-850 \mathrm{~nm}$ the refractive index of the metal $n_{M}$ weakly depends on $\lambda$, while $k_{M}$ monotonously increases with wavelength (Fig. 2 a,b). Moreover, the influence of the temperature regime is more

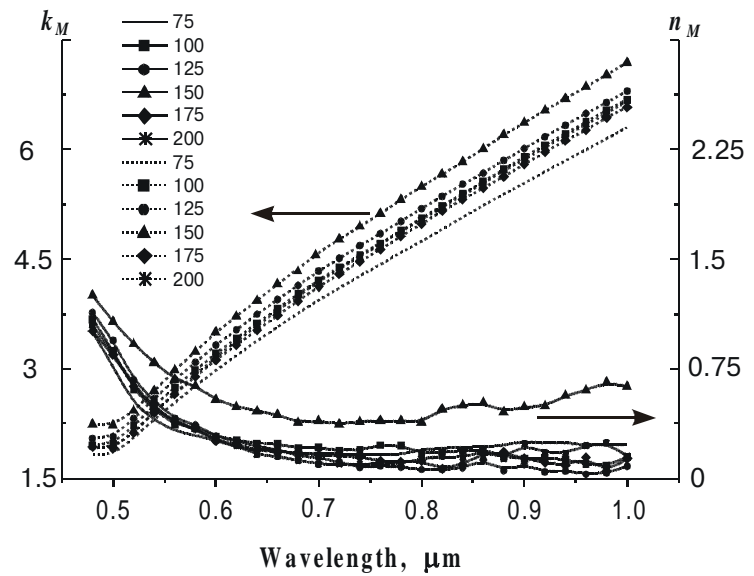

Fig. 2. Calculated from transmission and reflection spectra $n_{M}$ and $k_{M}$ values for thin gold films annealed (a) and evaporated onto hot substrates (b) at different temperatures. 


\section{B.A. Snopok et al.: Optical biosensors based on the surface plasmon resonance}

Table 1. Gold film optical constant for different anneling $\left(T_{A}\right)$ and substrate $\left(T_{S}\right)$ temperatures at $\lambda=630 \mathrm{~nm}$ as obtained from transmission/reflection measurements

\begin{tabular}{cccccccccccc}
\hline \hline $\begin{array}{c}\text { Temperature, } \\
{ }^{\circ} \mathrm{C}\end{array}$ & $T_{S}$ & $T_{S}$ & $T_{S}$ & $T_{S}$ & $T_{S}$ & $T_{S}$ & $T_{S}$ & $T_{A}$ & $T_{A}$ & $T_{A}$ & $T_{A}$ \\
\hline$n_{M}$ & 20 & 75 & 100 & 125 & 150 & 175 & 200 & 80 & 120 & 150 & 200 \\
\hline$k_{M}$ & 0.383 & 0.216 & 0.262 & 0.251 & 0.489 & 0.235 & 0.222 & 0.330 & 0.306 & 0.180 & 0.233 \\
\hline \hline
\end{tabular}

essential for the $k_{M}(\lambda)$ than for the $n_{M}(\lambda)$ dependence in the whole spectral range.

Optical parameters $\left(n_{M}\right.$ and $\left.k_{M}\right)$ for different annealing temperature and substrate temperature at $\lambda=630 \mathrm{~nm}$ are listed in Table 1. It is seen that principally different tendencies are observed in the gold film absorption coefficient in the range up to $150^{\circ} \mathrm{C}$ : while in the annealed films $k_{M}$ increases with temperature, for the samples deposited on the hot substrates $k_{M}$ decreases. Simultaneously, the absolute values of $k_{M}$ for the annealed films are in average by $0.1-0.6$ smaller as compared to the ones deposited on the hot substrates. Dependencies of the refraction index are also different: they are almost independent on temperature and decrease with the annealing temperature, respectively.

As it was shown above, to increase the sensitivity of the SPR transducer, one should diminish $k_{M}$. Thus, in view of the maximum sensitivity of the system the samples deposited at room temperature and annealed are more preferable than those deposited on the hot substrates. It should be emphasized that the lowest $k_{M}$ value is observed at the low temperature annealing in the temperature range $\sim 120-150^{\circ} \mathrm{C}$, that provides, as it was shown earlier [9], the optimum surface structure for the formation of self-assembled monolayers.

\subsection{X-ray study of the structure of thin gold films}

Fig. 3 (a) exhibits the diffraction spectra of the gold films deposited at the substrate temperature $T_{S}=20^{\circ} \mathrm{C}(1)$ and annealed at various temperatures $T_{A}(2-80,3-120$, $4-150,5-200,6-250$, and $7-300^{\circ} \mathrm{C}$, respectively). The lowest panel exhibits the standard spectrum of the polycrystalline gold [47]. These data evidence that the films have the polycrystalline structure with the preferential orientation of crystallites along $<011>$. The increase of the annealing temperature leads to the increase of the $<022>$ peak intensity and simultaneous decrease of other peaks intensities. This is the evidence of the disordering of the film.

The behavior of the wide maximum at the angles $14-32^{\circ}$ that does not correspond to the polycrystalline gold is especially interesting. This maximum can be ascribed to the presence of some quasiamorphous phase in the film with the crystallite sized 5-10 E. With the increase of the annealing temperature the intensity of the peak increases and after the annealing at $150^{\circ} \mathrm{C}$ (curve 4 ) in the region of small angle one more maximum appears that is also observed at higher annealing temperatures, while the right maximum disappears. This effect can be caused by the increase of nanocrystallite sizes in the quasiamorphous phase.

Both above effects decrease the inhomogeneity of the film and can lead to the decrease of the scattering and, consequently, of the thin film exctinction coefficient. At the same time, temperatures higher than $150^{\circ} \mathrm{C}$ stimulate formation of the phase with increased sizes of crystallites and causes the opposite effect.

Fig. 3 (b) exhibits the diffraction spectra of gold films deposited at various substrate temperatures $(1-75,2-$ $100,3-125,4-150,5-175,6-200^{\circ} \mathrm{C}$, respectively). Similarly to the first case, the gold film has a polycrystalline structure, but the preferable direction of their orientation is $\langle 111\rangle$. With the increase of the substrate temperature the degree of the disorder in the film increases (the intensity of the $<111>$ reflection peak increases as compared to other reflection peaks). In the low angle range there is also a wide maximum that is probably related to the presence of the gold crystallites of very small sizes (quasiamorphous phase). No changes of the position and the intensity of this maximum are observed at the increase of the substrate temperature.

It should be noted that reflection peaks $<022>$ and $<113>$ are asymmetric at low substrate temperatures, while at the temperatures 175 and $200^{\circ} \mathrm{C}$ these peaks are doublets (curves 5 and 6). Observation of two peaks evidences the presence of the phase of some other substance in the gold film. Since in our scheme we use chromium as an intermediate layer to improve the adhesion of gold on the glass the additional peaks are, most probably, related to the solid solution of chromium in gold. With the increase of the substrate temperature the intensity of the diffusion processes increases and, correspondingly, the content of chromium in the bulk increases, too; this process leads to the emergence of two peaks at sufficiently high substrate temperatures. The presence of the solid solution of chromium in gold probably leads also to the different $k_{M} v s$. temperature dependence in these samples (Table 1.).

\subsection{Topographic peculiarities of thin gold films}

The peculiarities of thin gold films relief generally correlate with the results of X-ray diffraction study. Surface images obtained using atomic force microscopy evidence 


\section{B.A. Snopok et al.: Optical biosensors based on the surface plasmon resonance}
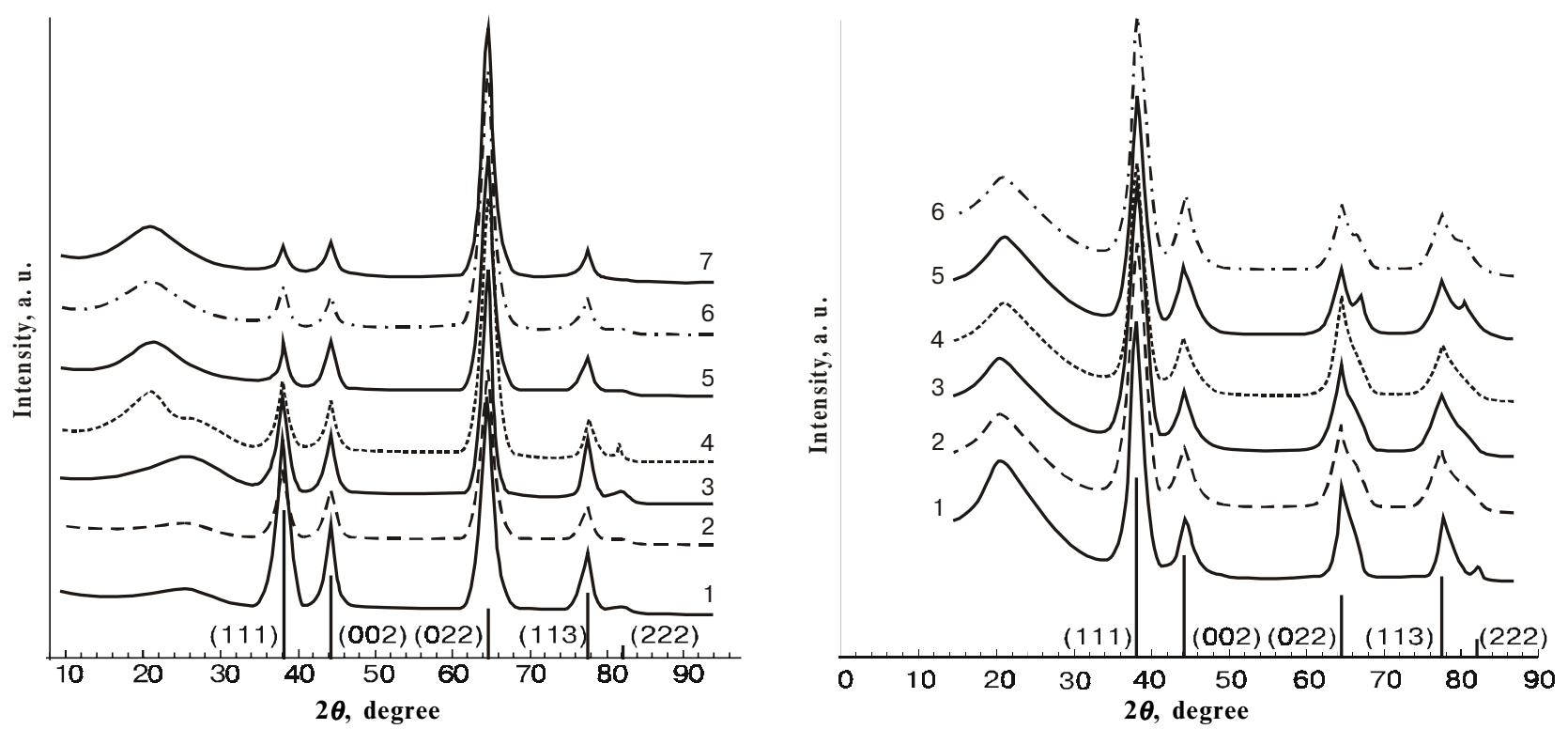

Fig. 3. X-Ray diffraction patterns for thin gold films annealed at temperatures (a): $1-20,2-80,3-120,4-150,5-200,6-250$, $7-300^{\circ} \mathrm{C}$; and (b) evaporated onto substrates kept at temperatures: $1-75,2-100,3-125,4-150,5-175,6-200^{\circ} \mathrm{C}$.

that the surface is not atomically smooth. The average diameter of seeds lies in the range from 20 to $30 \mathrm{~nm}$ for films deposited at $20^{\circ} \mathrm{C}$ and increases up to $\mathrm{ca} 120-150 \mathrm{~nm}$ and $c a 170-200 \mathrm{~nm}$ for the films annealed at $200^{\circ} \mathrm{C}$ and those deposited on the hot substrate at the same temperature, respectively. Root-mean-square roughness non-monotonously changes with the temperature starting with $\sim 0.93 \mathrm{~nm}$ for unannealed samples: at first it grows to $1.1 \mathrm{~nm}$ at $80^{\circ} \mathrm{C}$, then drops to $0.84 \mathrm{~nm}$ at $120^{\circ} \mathrm{C}$, then increases at $150^{\circ} \mathrm{C}$ to $0.95 \mathrm{~nm}$, and diminishes to 0.68 at $200^{\circ} \mathrm{C}$. These results correlate with the literature data that emphasize the non-regular variation of the surface roughness under the influence of the low temperature annealing of thin polycrystalline films [48]. For the films deposited on the hot substrates the mean square roughness is $1.5 \mathrm{~nm}, 1.97 \mathrm{~nm}, 1.5 \mathrm{~nm}, 2.57 \mathrm{~nm}, 4.4 \mathrm{~nm}$ and $5.04 \mathrm{~nm}$ for the temperatures $75,100,125,150,175$, and $200^{\circ} \mathrm{C}$, respectively. Thus, in this case the mean square roughness monotonously increases with the temperature increase up to $125^{\circ} \mathrm{C}$ and higher that evidences that the three-dimensional aggregates growth prevails as compared to layer-by-layer growth.

As we have shown earlier at the annealing of thin films in the range $120-140^{\circ} \mathrm{C}$ the phase transition caused by the recrystallization processes occurs; it leads to the formation of the crystallites of large sizes. Note, please, that for the samples deposited on the hot substrate at $T_{S}>150^{\circ} \mathrm{C}$ the sharp increase of the average crystallite size is also observed. Thus, at the temperatures in the range $120-140^{\circ} \mathrm{C}$ some fundamental processes of metal growth/reorganization are activated in thin films, and they control the structure formation independently on the deposition method and prevailing orientation.

It should be noted that root-mean-square roughness values calculated using the light scattering data and atomic force microscopy data do not correlate. At the same time, there is a correlation between the scattering intensity (Fig. 4) and optical parameters of the metal film. Moreover, the scattering of light is caused by topographic peculiarites of thin metal films and being minimal at annealing temperatures close to $120^{\circ} \mathrm{C}$.

\subsection{Surface plasmon resonance in thin gold films}

The above discussed characteristics of the effective optical constants of thin gold films, their morphological and topographical features lead to the conclusion that the increase of temperature up to $\sim 120-130^{\circ} \mathrm{C}$ does not essentially vary the crystallite sizes; it only changes the structure (the texture becomes complicated) and the sur-

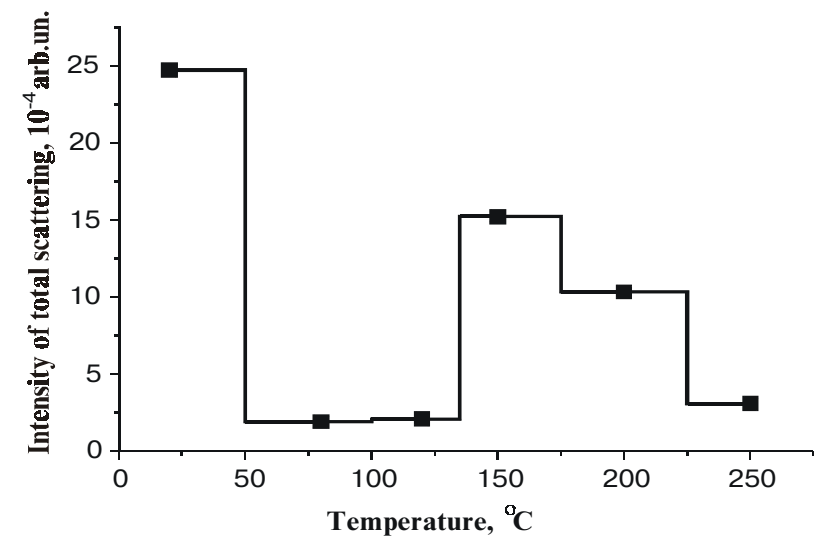

Fig. 4. Total integrated scattering (for normal incidence of light) for thin gold films annealed at different temperatures. 


\section{B.A. Snopok et al.: Optical biosensors based on the surface plasmon resonance}

face relief (the low-scale roughness decreases). At the temperatures above $150^{\circ} \mathrm{C}$ due to the recrystallization processes the average size of the crystallites increases; this can cause the worsening of the operating characteristics of thin film SPR transducer (due to the scattering increase, etc.). But, prior to discuss the dependence of operation characteristics of SPR transducers on the fabrication temperature regimes, let us analyze the influence of the substrate type (quartz, glass), chromium adhesive layer and metal film thickness on the character and the position of SPR curves.

The influence of the substrate type. As seen from Fig. 5,a the decrease of the refractive index of substrate $n_{S}$ from 1.51 for glass to 1.46 for quartz shifts the SPR curve $\left(\approx 2.2^{\circ}\right)$ to the large angles range in accordance with the calculated value $\left(\approx 2.14^{\circ}\right)$. The larger roughness that is characteristic for the glass substrates ( $c a .2 \mathrm{~nm})$ as compared to quartz ( $\mathrm{ca} .1 .1 \mathrm{~nm}$ ) leads to the increase of $R_{\text {min }}$ (from 0.096 to 0.129 ) and halfwidth of the curve $\Delta R$ $\left(\approx 0.41^{\circ}\right)$ in accordance with theoretical considerations discussed above. Therefore, in view of application of our structures in SPR transducer the quartz substrates are preferable.

Adhesive chromium layer. Fig. 5,b shows the angular dependencies of the reflection from the structure quartz/metal film/air; in this case the metal film consists of the gold film ca. $45 \mathrm{~nm}$ and subsequently deposited $\mathrm{Cr}$ and $\mathrm{Au}(45 \mathrm{~nm})$. The chromium layer has an island needle-like structure with the effective thickness $c a$. $1 \mathrm{~nm}$. As seen from the Fig. 5,b the curves almost coincide near the minimum (the shift is about $0.06^{\circ}$ ). At the same time, the curve that corresponds to the structure that includes the adhesion chromium layer exhibits larger halfwidth $\left(\approx 0.5^{\circ}\right)$ and smaller reflection values in the TIR range and at large angles. The refractive index values measured in these films by ellipsometry method are $0.23+i 3.45$ $\left(d_{M}=44.94 \mathrm{~nm}\right)$ and $0.3+i 3.42\left(d_{M}=44.94 \mathrm{~nm}\right)$ for $\mathrm{Au}$ and $\mathrm{Cr}+\mathrm{Au}$ systems, respectively. In agreement with the above discussion, these are the changes in $n_{M}$ that correlate with the observed changes of SPR curves. Thus, the presence of the chromium adhesion layer increases the effective value of the real part of the refraction index $n_{M}$ of the metal layer, if the calculations are carried out in the one-layer model (i.e., the system $\mathrm{Cr}+\mathrm{Au}$ is considered as a single layer with effective optical constants). In the framework of this model, the calculated gold film parameters are: $\mathrm{Au}-(0.162+i 3.383), \mathrm{Cr}+\mathrm{Au}-(0.167+i 3.429)$; they totally agree with the above conclusions.

Metal film thickness. As it was noted above, the thickness of the metal film is an important factor that influences the characteristics of SPR transducer. The dependencies in Fig. 5,c support the above statements that both decrease and increase of $d_{M}$ essentially change the shape of the SPR curve: if $d_{M}$ is larger than the optimum value, the $R_{\min }$ increases, if $d_{M}$ is larger, then the $\Delta R$ increases. Especially interesting is the fact that influence of the annealing temperature depends on the film thickness. E.g., if the film thickness is less than the optimum one the annealing causes the shift of the SPR curve towards large
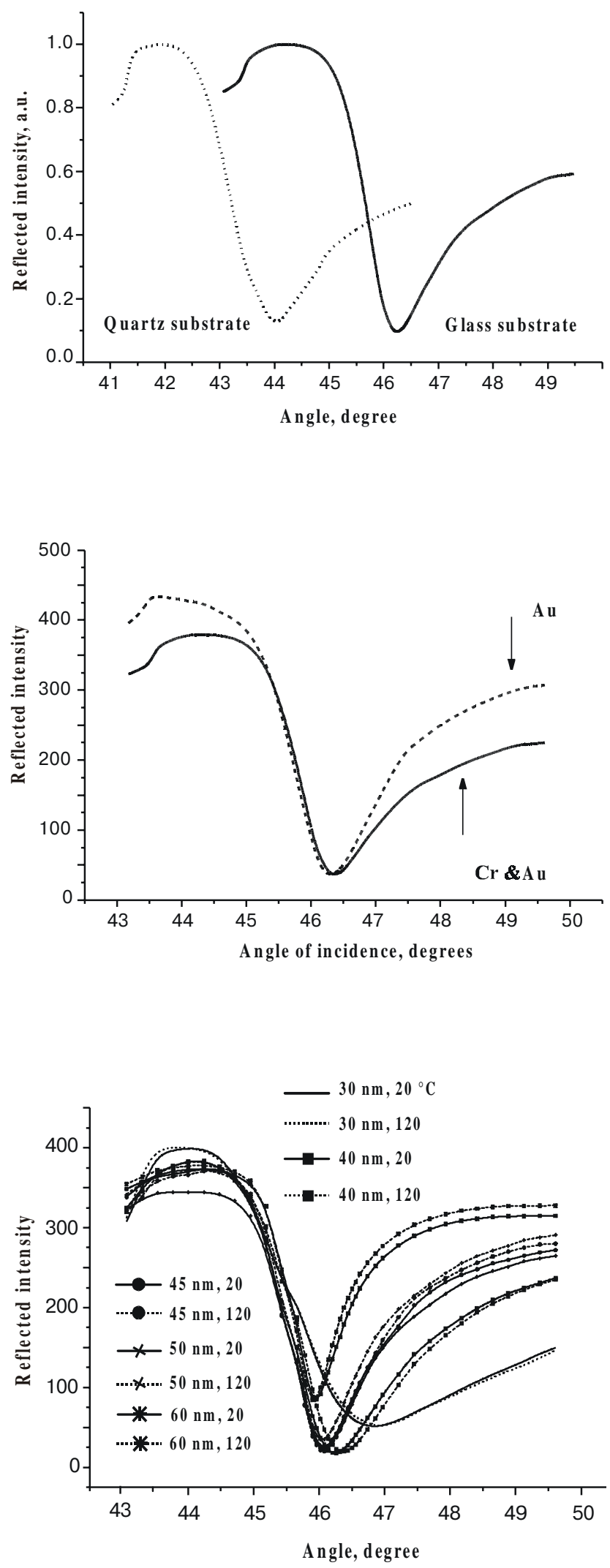

Fig. 5. Comparison of SPR curves for different materials of support plates (a), presence of adhesive Cr layer (b) and the effect of annealing on films with different thicknesses (c). 


\section{B.A. Snopok et al.: Optical biosensors based on the surface plasmon resonance}

angles, if $d_{M}$ is larger than the optimum the curve shifts towards small angles; in the vicinity of the optimum thickness $(\sim 45 \mathrm{~nm})$ the shift is the least sizable.

Dependencies of shape SPR curves on the value and the character of the thermal regimes of transducer fabrication. While the influence of the temperature is more pronounced when the film thicknesses are far from optimum value it is the optimized structure rather interesting in view of its practical applications. Fig. 6 a,b shows the resonance SPR curves for the samples annealed (a) and obtained (b) at various temperatures. Comparison of the curves on Figs $6 \mathrm{a}$ and $\mathrm{b}$ leads to the conclusion that the regime of transducer fabrication, that implies gold deposition on the hot substrate, is more sensitive to substrate temperature variations than the regime that implies annealing. In the first case, the step-like variation of $\Delta R$ and quasistationary value of $\theta_{S P R}$ are observed in the temperature range $120-150^{\circ} \mathrm{C}$. Changes of the reflection intensity in the range of the transition to TIR and at large angles correlate with each other and are characterized by the monotonous parabolic dependence with the minimum in the range $130-150^{\circ} \mathrm{C}$. This dependence evidences that at these temperatures of the film refractive index $n_{M}$ reaches the largest value, i.e., the film has the most efficient package from the structural point of view. The steplike variation of $R_{\min }$ evidences the essential decrease of $n_{M}$ at the temperature $150^{\circ} \mathrm{C}$; it can be caused by the formation of another type of structures at these temperatures (see the emergence of the new peak in X-ray spectra, Fig. 3.). The nonmonotonous variation of $\theta_{S P R}$ can be caused by the same reason, i.e., by the dependence of $k_{M}$ on the structure parameters.

For the films deposited on the cold substrate and annealed at various temperatures one does not see such strong dependencies as in the previous case. The SPR curves are similar. At the same time, the samples annealed at $120^{\circ} \mathrm{C}$ are characterized by the minimum values of $R_{\min }$ and $\Delta R$ as well as by maximum reflectance in the TIR region that provides the best operating parameters of the SPR transducers based on the films. As it was noted above, it were these samples where the topography parameters evidenced the minimum light scattering of the films. The dependence of the integral scattering in thin gold films at SPR excitation conditions (Fig. 7.) evidence that radiative losses of the energy of the surface polariton states are also minimum at the annealing temperature $c a$ $120^{\circ} \mathrm{C}$. Thus, the morphological, topographical and, as a consequence, optical characteristics of thin gold films deposited at the substrate temperature $20^{\circ} \mathrm{C}$ and annealed at $120^{\circ} \mathrm{C}$ have optimum characteristics for SPR transducer applications.

Time stability of the SPR transducer parameters. Another important feature of SPR transducers is their time stability that depends mostly on the stability of gold film characteristics. Table 2 lists the values for the transducers based on the films deposited at $20^{\circ} \mathrm{C}$, annealed at 120 and $150^{\circ} \mathrm{C}$, and the films deposited at 125 and $150^{\circ} \mathrm{C}$. It is seen that transducers using the films deposited on the hot substrate do not relax during the first day after the deposition. At the same time, the character of the relaxation during the following days is the same for all samples and can be caused by the adsorption of organic, mostly, sulfur-containing molecules on the surface of the film from the gas phase. Some relaxation of the annealed film during the first day after the deposition is probably caused by the mechanical strain relaxation [34].

In spite of high chemical stability of gold, its thin films tend to change their physical and chemical characteristics during long-term operation in liquids. To explain the changes of the optical parameters of the films, one should take into account both the changes of the upper transient layer and structure transformations in the bulk of polycrystalline films. E.g., the diffusion along the seeds boundaries in the polycrystalline film probably causes the distortion of the lattice bonds on the interface changing in such manner the strength and character of intermolecular interactions and, as a result, the parameters of intercrystallite contacts [49].

To understand the mechanism of the chemical «aging» of thin films, the detailed study using differential IR and photoelectronic spectroscopy methods should be carried out, but, analyzing the character of SPR curves parameters variation, one can make some suggestions about correponding mechanisms. Due to the polycrystalline structure of our thin gold films, the water from the reactive medium penetrates into the intercrystallite space. Naturally, this can cause the change of the optical parameters of gold as compared to single crystalline samples. Therefore, taking into account low films thicknesses and, hence, technological problems of poreless layers

Table 2. Resonance angle values $\theta_{S P R}$ changing with time

\begin{tabular}{c|ccccc}
\hline \hline \multirow{2}{*}{$\begin{array}{c}\text { Conditions } \\
\text { of sample fabrication }\end{array}$} & \multicolumn{4}{|c}{ Resonance angle values (in arc degrees) } \\
\cline { 2 - 6 } & 1 day & 3 day & 7 day & 30 day & 100 day \\
\hline Without annealing & 43,58 & 43,69 & 43,69 & 43,75 & 43,85 \\
\hline Annealing temperature $120^{\circ} \mathrm{C}$ & 43,69 & 43,75 & 43,75 & 43,85 & 43,90 \\
\hline Annealing temperature $150^{\circ} \mathrm{C}$ & 43,63 & 43,69 & 43,69 & 43,75 & 43,80 \\
\hline Substrate temperature $125^{\circ} \mathrm{C}$ & 43,69 & 43,69 & 43,69 & 43,75 & 43,80 \\
\hline Substrate temperature $150^{\circ} \mathrm{C}$ & 43,69 & 43,69 & 43,75 & 43,80 & 43,80 \\
\hline \hline
\end{tabular}


B.A. Snopok et al.: Optical biosensors based on the surface plasmon resonance
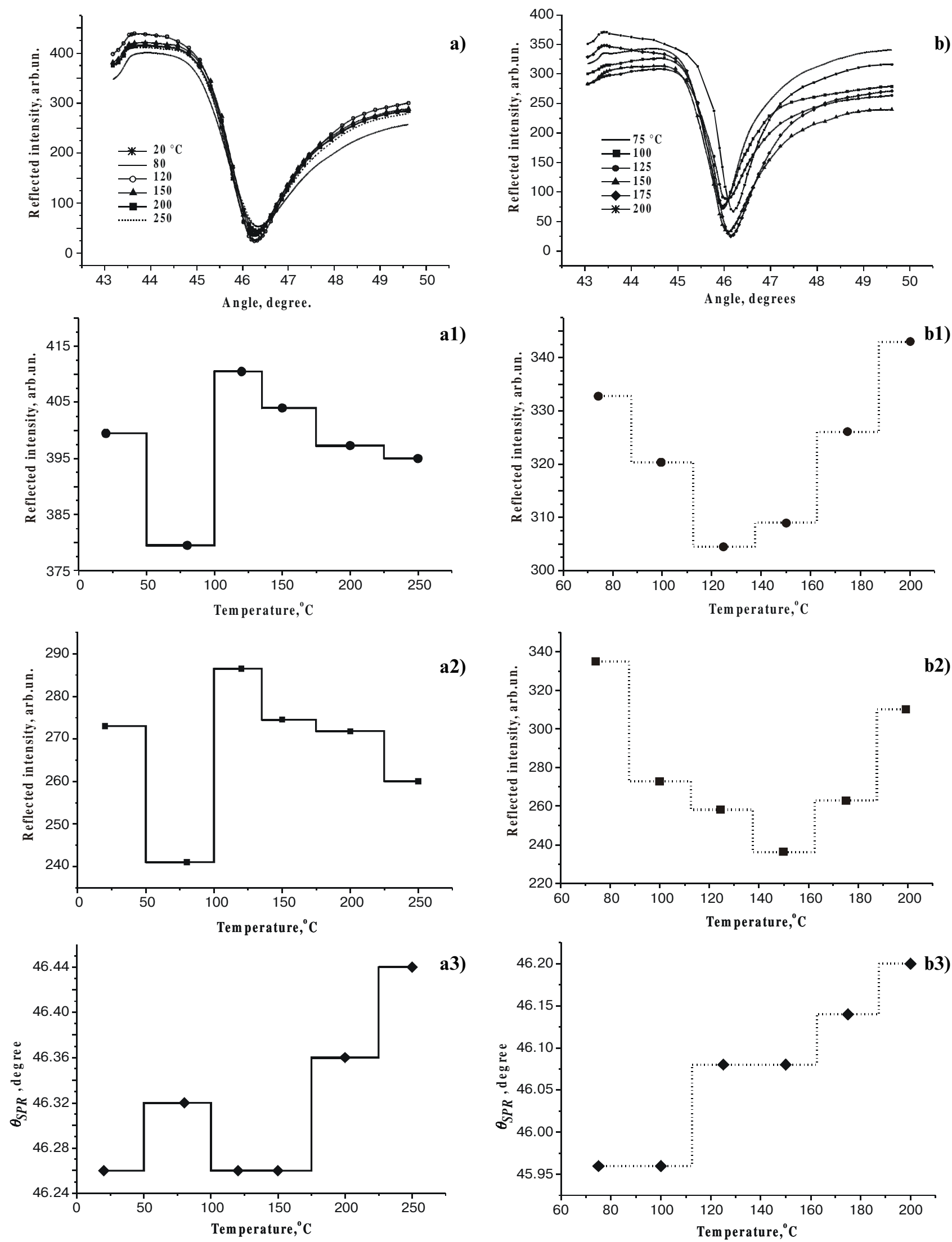

Fig. 6. SPR curves versus annealing temperature (a) and temperature of the support plate under evaporation (b). Figs (a1)/(b1) and (a2)/ (b2) represent sections of the primary SPR sets before $\left(44.83^{\circ}\right)$ and after $\left(48.89^{\circ}\right)$ the curve minimum, correspondingly and (a3)/(b3) position of the minimum versus temperature. 


\section{B.A. Snopok et al.: Optical biosensors based on the surface plasmon resonance}

Table 3. Gold film optical constant for different anneling $\left(T_{A}\right)$ and substrate $\left(T_{S}\right)$ temperatures at $\lambda=630 \mathrm{~nm}$ as obtained from SPR measurements

\begin{tabular}{cccccccccccc}
\hline \hline $\begin{array}{c}\text { Temperature, } \\
{ }^{\circ} \mathrm{C}\end{array}$ & $T_{S}$ & $T_{S}$ & $T_{S}$ & $T_{S}$ & $T_{S}$ & $T_{S}$ & $T_{S}$ & $T_{A}$ & $T_{A}$ & $T_{A}$ & $T_{A}$ \\
\hline$n_{M}$ & 20 & 75 & 100 & 125 & 150 & 175 & 200 & 80 & 120 & 150 & 200 \\
\hline$k_{M}$ & 0.167 & 0.074 & 0.081 & 0.082 & 0.152 & 0.119 & 0.08 & 0.178 & 0.179 & 0.154 & 0.167 \\
\hline \hline
\end{tabular}

fabrication, one comes to the conclusion that to obtain reliable sensitive elements for the operation in water solutions, it is not sufficient to use only thermal regimes of the gold films fabrication. To prevent the «aging» of thin polycrystalline gold films the self-assembled thiol monolayers can be used [50].

\subsection{Macroscopic model of thin gold films}

The optical model of thin gold films should account for their spatially inhomogeneous structure, i.e., for the main features that influence light propagation and reflection both in the bulk and on the surface.

In view of the important role of the surface roughness in the experiments with surface plasmon excitation, we will describe the real polycrystalline gold film by the two-layer model: the spatially homogeneous film with the optical parameters close to those in bulk samples and the spatially inhomogeneous layer caused by the surface roughness. Since in the SPR theory only the optically homogeneous parallel layers are described, we will use a simplified approach [18] and will substitute the rough «surface layer» with an equivalent layer with parallel surfaces and the thickness equal to the mean square root roughness value. In this model the optical parameters of the resulting system are determined using the optical parameters of the material and surrounding medium in the

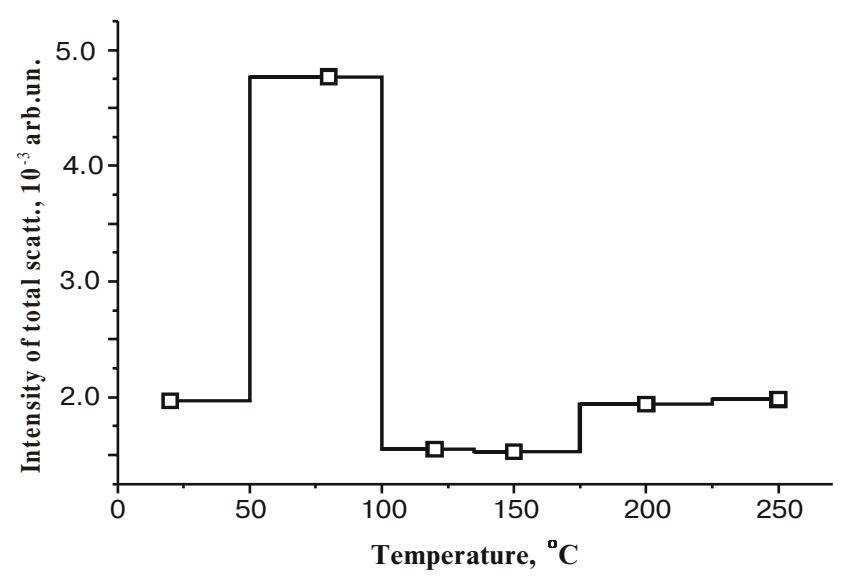

Fig. 7. Total integrated radiation scattering of surface plasmons versus temperature. The measurements were made at the angle corresponding to the minimum of reflected intensity. approximation of additive refraction in accordance with the model of the effective optical parameters of inhomogeneous media.

Approximation of experimental data both in the framework of monolayer and bilayer models showed that addition of the second layer is not necessary. This evidences that contribution of a rough layer can be taken in to account through effective parameters of the monolayer model at least (for investigations in air).

Optical parameters obtained using numerical simulation of the experimental curves in the single layer are listed in Table 3. It is seen that in the case of the samples deposited at $20^{\circ} \mathrm{C}$ and annealed at various temperatures the whole plasmon resonance curve is pretty well described when the parameters of layer are close to those of the bulk material. In the case of samples deposited on the hot substrate, one can observe the same behaviour. $n_{M}$ and $k_{M}$ temperature dependences correspond well to results obtained using transmission and reflection spectra.

Analysis of the SPR dependencies in the framework of the classical macroscopic approach for the samples obtained by different technological regimes showed that using the adequate model of the thin layer one can correctly describe the processes of the interface. SPR spectroscopy monitor the variation of the surface state during the adsorption and to characterize the multi-layer structure.

\section{Conclusions}

Effective operation of sensor systems depends on two factors: i) biochemical functioning of the receptor layer, ii) sensitivity and stability of the physical transducer. While a lot of efforts was done to achieve the sufficient specific sensitivity of the sensor, the problem of the optimization of the parameters of thin film PT of the one-electron type has not been studied yet.

In the present paper, the ways to improve sensitivity and stability of the plasmon resonance transducer and to optimize its operating characteristics are analyzed in the framework of the complex approach. It is shown that the simple procedure of the low temperature annealing (at $\approx 120^{\circ} \mathrm{C}$ ) provides optimum parameters of the thin gold film (where the plasmon vibrations occur) in view of its application as a physical transducer in optical biosensors. The morphological and topographic features of the thin films structure were analyzed using X-rays diffrac- 


\section{B.A. Snopok et al.: Optical biosensors based on the surface plasmon resonance}

tion atomic force microscopy, respectively. The influence of the structure features on the optical characteristics (transmission, reflection, scattering) and optoelectronic processes on the interface ( SPR effects) was analyzed. The ways for optimization of SPR transducer were proposed, and stable high sensitive SPR elements used in the device «PLASMON-4M» elaborated in the Institute of Semiconductor Physics (NAS of Ukraine) were fabricated. These devices provide optimum conditions for the formation of self-assembled recepting structures on the thin film surface.

\section{References}

1. P. Bongrand, Ligand-receptor interactions // Rep. Prog. Phys. 62, pp.921-968 (1999).

2. M. J. O`Brien II, S. R. J. Brueck, V. H. Perez-Luna, L. M. Tender, G. P. Lopez, Spr biosensors: simultaneously remuving thermal and bulk-composition effects // Biosensors and Bioelectronics 14, pp. 145-154 (1999).

3. J.J.Ramsden, Optical biosensors // J. Mol. Recognition 10, pp. 109-120 (1997).

4. L.S.Jung, C.T.Campbell, T.M.Chinowsky, M.N.Mar, S.S.Yee, Quantitative interpretation of the response of surface plasmon resonance sensors to adsorbed films // Langmuir 14, pp. 5636-5648 (1998).

5. L.-H. Guo, J. S. Facci, G. McLendon, R. Mosher, Effect of gold topography and surface pretreatment on the self-assembly of alkanethiol monolayers // Langmuir 10, pp. 4588-4593 (1994).

6. Y. Okawa, V. Nagano, S. Hirota, H. Kobayashi, T. Ohno, M. Watanabe, Tethered mediator biosensor. Mediated electron transfer between redox enzyme and electrode via ferrocene anchored to electrode surface with long poly(0xyethylene) chain // Biosensors and Bioelectronics 14, pp. 229-235 (1999).

7. G. Decher, Layered nanoarchitectures via directed assembly of anionic and cationic molecules, Chap. 14 in Comprehensive supramolecular chemistry, Eds. G. Abberti, T. Bein, pp. 507-530, Elsevier Science Ltd (1996).

8. M.D. Mowery, H. Menzel, M. Cai, C.E. Evans, Fabrication of monolayers containing internal molecular scaffolding: effect of substrate preparation // Langmuir 14, pp. 5594-5602 (1998).

9. B.A. Snopok, P.E. Strizhak, E.V. Kostyukevich, V. Serebriy, S.I. Lysenko, P.E. Shepeliavii, S.L. Priatkin, S.A. Kostyukevich, Yu.M. Shirshov, E.F. Venger, Interfacial architecture on the fractal support: polycrystalline gold films as support for selfassembling monolayers // Semiconductor Physics, Quantum Electronics and Optoelectronics 2(3), pp. $86-97$ (1999).

10. A.F. Collings, F. Caruso, Biosensors: recent advances // Rep. Prog. Phys. 60, pp.1397-1445 (1997).

11. Z. Salamon, H.A. Macleod, G. Tollin, Surface plasmon resonance spectroscopy as a tool for investigating the biochemical and biophysical properties of membrane protein systems // Biochimica et Biophysica Acta 1331, pp. 117-152 (1997).

12. M.P. Byfield, R.A. Abuknesha, Biochemical aspects of biosensors // Biosensors and Bioelectronics 9, pp. 373-400 (1994).

13. V.C. Millot, F. Martin, D. Bousquet, B. Sebille, Y. Levy, A reactive macromolecular matrix for protein immobilization on a gold surface. Application in surface plasmon resonance // Sensors and Actuators B 29, pp. 268-273 (1995).

14. B. Klee, G.L. Duveneck, P. Oposzlan, M. Ehrat, H.M. Widmer, A model system for the development of an optical biosensor based on lipid membranes and membrane-bound receptors // Sensors and Actuators B 29, pp. 307-311 (1995).

15. B. Liedberg, I. Lundstrom, E. Stenberg, Principles of biosensing with an extended coupling matrix and surface plasmon resonance // Sensors and Actuators B 11, pp. 63-72 (1993).
16. M. Manuel, B. Vidal, R. Lopez, S. Alegret, J. AlonsoChamarro, Determination of probable alcohol yield in musts by means of an SPR optical sensor// Sensors and Actuators B11, pp. 455-459 (1993).

17. C.E.H. Berger, J. Greve, Differential SPR immunosensing // Sensors and Actuators B 63, pp. 103-108 (2000).

18. B.A. Snopok, K.V. Kostyukevich, O.V. Rengevych, Yu.M. Shirshov, E.F. Venger, A biosensor approach to probe the structure and function of the adsorbed proteins: fibrinogen at the gold surface // Semiconductor Physics, Quantum Electronics and Optoelectronics 1(1), pp. 121-134 (1998).

19. H.E. de Bruijn, R.P.H. Kooyman, J. Greve, Choice of metal and wavelength for surface-plasmon resonance sensors: some considerations // Applied Optics. 31(4), pp. 440-442 (1992).

20. A. Zangwill, Physics at surfaces, Cambridge University Press (1998).

21. W. Knoll, Guided wave optics for the characterization of polymeric thin films and interfaces, Chap. 13 in Handbook of optical properties, v.1, Thin films for optical coatings, Eds. R. E. Hummel, K. H. Guenther, CRC Press, Inc. (1997).

22. N.L. Dmitruk, V.G. Litovchenko, V.L. Strizhevskyj, Surface polaritons in semiconductor and dielectric, Naukova dumka, Kiev (1989), (in Russian).

23. G. Boisde, A. Harmer, Chemical and biochemical sensing with optical fibers and waveguides, Artech House, Inc., Boston (1996).

24. X. Caide, S-F. Sui, Characterization of surface plasmon resonance biosensor // Sensors and Actuators B 66, pp. 174-177 (2000).

25. Surface polaritons, Eds. V. M. Agranovich, D. L. Mellse, Nauka, Moscow (1985), (in Russian).

26. G.V. Beketov, Yu.M. Shirshov, O.V. Shynkarenko, V.I. Chegel, Surface plasmon resonance soectroscopy: prospects of superstrate refractive index variation for separate extraction of molecular layer parameters//x Sensors and Actuators B 48, pp. 432-438 (1998).

27. O.V. Rengevich, Yu.M. Shirshov, Yu.V. Ushenin, A.G. Beketov, Separate determination of thickness and optical parameters by surface plasmon resonance: accuracy consideration // Semiconductor Physics, Quantum Electronics \& Optoelectronics 2(2), pp. 28-35 (1999).

28. R.M.A. Azzam, N.M. Bashara // Ellipsometry and polarized light, North-Holland, Amsterdam (1977).

29. A.V. Rakov, Spectrofotometry thin semiconductor structures, Sovetskoe radio, Moscow (1975), (in Russian).

30. H. Raether, Surface plasmons on smooth and rough surfaces and on gratings, Springer-Verlad, Berlin (1985).

31. J.A. De Feijter, J. Benjamins, F.A. Veer, Ellipsometry as a tool to study the adsorption behaviour of polymers at the airwater interface // Biopolymers 17 pp. 1759 (1978).

32. M. Malmsten, Ellipsometry stadies of protein layers adsorbed at hydrophobic surfaces // Journal of colloid and interface science 166, pp. 333-342 (1994).

33. S.I. Lysenko, B.A. Snopok, E.V. Kostyukevich, V.A. Sterligov, Yu.M. Shirshov, Light scattering of self-assembled monolayers on the surface of polycrystalline gold // Optics and spectroscopy 90 (3), (2001), (in Russian).

34. M. Aguilar, A.I. Oliva, P. Quintana, J.L. Pena, Dynamic phenomena in the surface of gold thin films: macroscopic surface rerrangements // Surface Science 380, pp. $91-99$ (1997).

35. N. Kroo, W. Krieger, Z. Lenkefi, Z. Szentirmay, J.P. Thost, H. Walther, A new optical method for investigation of thin metal films // Surface Science 331-333, pp. 1305-1309 (1995).

36. E.V. Kostyukevich, B.A. Snopok, S.A. Zinio, Yu.M. Shirshov, I.N. Kolesnikova, E.N. Lugovskoi, New opto-electronic system based on the surface plasmon resonance phenomenon: application to the concentration determination of DD-fragment of fibrinogen // Proceedings of SPIE 3414, pp. 290-301 (1998).

37. A.N. Matveev, Optics, Vysshaj shkola, Moscow (1985), (in Russian). 


\section{B.A. Snopok et al.: Optical biosensors based on the surface plasmon resonance}

38. J.H. Weaver, Optical properties of metals, in Handbook on chemistry and physics, Eds. R.C.W. Weas, Chemical Rubber Publishing Company,USA (1986).

39. P.B. Johnson, R.W. Christy, Optical constant of the noble metals // Phys.Rev. B 6(12), pp.4370-4379 (1972).

40. E.D. Palik, Handbook of optical constants of solids, Academic Press, Orlando-Sandiego (1985).

41. Z. Salamon, H.A. Macleod, G. Tollin, Surface plasmon resonance spectroscopy as a tool for investigating the biochemical and biophysical properties of membrane protein systems. I. Theoretical principles // Biochimia et Biophysica Acta 1331, pp. 117-129 (1997).

42. S.A. Kovalenko, R.D. Fedorovych, Optical properties of thin gold films // Semiconductor Physics, Quantum Electronics and Optoelectronics 3 (3), pp. 383-388 (2000).

43. S.A. Kovalenko, Optical properties of thin metal films // Semiconductor Physics, Quantum Electronics and Optoelectronics 2(3), pp. 13-20 (1999).
44. R. Abermann, H.P. Martinz, R. Kramer, Thermal effects during the deposition of thin films // Thin Solid Films 70(1), pp. 127-137 (1980)

45. G. Dumpich, Quantitative analysis of the growth of gold films on carbon layers // Thin Solid Films 127, pp. 323-335 (1985).

46. A. Piegari, E. Masetti, Thin film thickness measurement: a comparison of various techniques // Thin Solid Films 124, pp. 249-257 (1985).

47. L.I. Mirkin, Handbook on X-ray structure analysis polycrystals, Literatyra, Moscow (1961), (in Russian).

48. J.M. Elson, Theory of light scattering from a rough surface with an anhomogeneous dielectric permittivity // Physical Review B 30, pp. 5460-5480 (1984).

49. I.M. Poletina, Intercrystalline adsorption of impurities and metals destruction, Nauka, Novosibirsk (1988).

50. H. Ron, S. Matlis, I. Rubinstein, Self-assembled monolayers on oxidized metals. 2. Gold surface oxidative pretreatment. Monolayer properties and depression formation // Langmuir 14, pp. 1116-1121 (1998) 